\title{
HELMINTOS GASTRINTESTINAIS DE Chelonia mydas (TARTARUGAS-VERDES) RESGATADAS NO LITORAL SUL DE SÃO PAULO, BRASIL
}

(Helminths of Chelonia mydas (green sea turtles) rescued on the South Coast of São Paulo, Brazil)

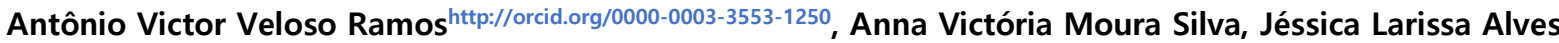 \\ Dias, Éllen Araújo de Deus, Vanessa Paulino da Cruz Vieira
}

Instituto Federal do Norte de Minas Gerais (IFNMG), Salinas, Minas Gerais

*Corresponding author: antonioifnmg@yahoo.com.br

Editora: Julia Arantes Galvão

RESUMO: Objetivou-se realizar o levantamento de helmintos gastrintestinais de Chelonia mydas (tartarugas-verdes) resgatadas no litoral sul de São Paulo, Brasil. Para isso, foram analisados 118 laudos de exames coproparasitológicos e anatomopatológicos realizados pelo Instituto Biopesca entre 2018 e 2019, oriundos do Sistema de Informação de Monitoramento da Biota Aquática, obtendo-se o número de animais parasitados, espécies de helmintos gastrintestinais encontrados, o órgão parasitado e a presença de mono ou poliparasitismo. Os índices ecológicos parasitários de prevalência, intensidade média e abundância média foram calculados. Os resultados revelaram 102 animais albergavam endoparasitos, com prevalência de $86,44 \%$ de C. mydas parasitadas. Os órgãos mais acometidos foram intestino delgado (63,06\%) e estômago (63,06\%). Foram identificadas 13 espécies da Classe Trematoda e as maiores prevalências foram observadas para os helmintos Cricocephalus albus (75,49\%), Metacetabulum invaginatum (42,15\%) e Pronocephalus obliquus (28,43\%). O helminto mais abundante foi C. albus $(11,86)$, seguido por $M$. invaginatum $(11,61)$ e $P$. obliquus $(4,50)$. A maior intensidade média encontrada foi da espécie $M$. invaginatum $(31,88)$, e depois para $P$. obliquus $(18,34)$ e para C. albus $(18,18)$. Entre as tartarugas parasitadas, $42,37 \%$ apresentavam monoparasitismo e 51,69\% apresentavam poliparasitismo, com associação entre duas até oito espécies de parasitos. Os helmintos gastrintestinais de Chelonia mydas resgatadas no litoral sul de São Paulo é constituída predominantemente de trematódeos, com uma elevada prevalência, intensidade e abundância média quando comparados à literatura existente.

Palavras-chave: parasitos; Chelonidae; marinhos.

ABSTRACT: The objective was to carry out a survey of gastrointestinal helminths of Chelonia mydas (green turtles) rescued on the south coast of São Paulo, Brazil. For this, 118 reports of coproparasitological and anatomopathological exams performed by the 
Biopesca Institute between 2018 and 2019, from the Aquatic Biota Monitoring Information System, were analyzed, obtaining the number of parasitized animals, species of gastrointestinal helminths found, the parasitized organ and the presence of mono or polyparasitism. The parasitic ecological indices of prevalence, average intensity and average abundance were calculated. The results revealed 102 animals housed endoparasites, with a prevalence of $86.44 \%$ of parasitized C. mydas. The most affected organs were the small intestine (63.06\%) and stomach (63.06\%). 13 species of the Trematoda Class were identified and the highest prevalences were observed for the helminths Cricocephalus albus (75.49\%), Metacetabulum invaginatum (42.15\%) and Pronocephalus obliquus (28.43\%). The most abundant helminth was C. albus (11.86), followed by $M$. invaginatum (11.61) and $P$. obliquus (4.50). The highest mean intensity found was of the species $M$. invaginatum (31.88), and then for P. obliquus (18.34) and for C. albus (18.18). Among the parasitized turtles, $42.37 \%$ had monoparasitism and $51.69 \%$ had polyparasitism, with an association between two to eight species of parasites. The gastrointestinal helminths of Chelonia mydas rescued on the south coast of São Paulo are predominantly trematodes, with a high prevalence, intensity and average mean when compared to the existing literature.

Keywords: helminths; Chelonidae; marine.

\section{INTRODUÇÃO}

Os répteis surgiram há cerca de 180 milhões de anos e povoam o planeta em ambientes aquáticos e terrestres. Ao decorrer de sua linha evolutiva as tartarugas marinhas sofreram diversas modificações que auxiliaram na sua sobrevivência no ambiente marítimo. Por exemplo, as vértebras reduziram, costelas se fusionaram formando o casco e as patas modificaram-se em nadadeiras (Cubas e Baptistotte, 2014). As tartarugas marinhas utilizam o ambiente terrestre somente para postura. São importantes componentes dos ecossistemas marinhos, estando presentes em todos os oceanos, mantendo o equilíbrio e participando de cadeias alimentares essenciais a vida de outras espécies, como peixes e crustáceos. As pressões ambientais de origem antrópica, degradando e explorando ecossistemas de forma exacerbante, contribuem para a condição de vulnerabilidade e extinção das tartarugas marinhas. As principais ações antropogênicas que atingem estes animais enquadram a caça proposital, a pesca incidental, consumo de ovos de maneira desenfreada, destruição de vegetação em áreas de postura e poluição dos mares (Spotila, 2004; Kamel et al., 2006). 
Cinco das sete espécies de tartarugas marinhas, consideradas os mais primitivos répteis existentes, são encontradas nas costas brasileiras. Sendo estas: a Caretta caretta (Linnaeus, 1758), popularmente conhecida como tartaruga-cabeçuda, Eretmochelys imbricata (Linnaeus, 1766) ou tartaruga-de-pente, a Lepidochelys olivácea (Eschscholtz, 1829 também conhecida como tartaruga-oliva, Dermochelys coriácea (Linnaeus, 1766) a tartaruga-de-couro, e a Chelonia mydas (Linnaeus, 1758) ou tartaruga-verde (Marcovaldi e Marcovaldi, 1999).

A C. mydas é um animal classificado como vulnerável no Brasil (Brasil, 2015) e internacionalmente tem o status de espécie ameaçada (IUCN, 2016). Doenças também são outro fator, como a fibropapilomatose e as parasitoses, que deixam as tartarugas extremamente debilitadas (Werneck e Silva, 2015). Os animais encalhados geralmente hospedam uma abundante e diversificada fauna de endoparasitos, que apresentam adaptação ao ambiente marinho e aos hábitos migratórios de seus hospedeiros (Greiner, 2013). Ressalta-se que ações antrópicas também podem influenciar no parasitismo das tartarugas, ao exercer pressão no ciclo de vida de várias espécies e modificando as relações existentes entre hospedeiros e parasitos (Silva et al, 2010).

De acordo aos estudos realizados por Klingenberg (1993), o parasitismo pode afetar esses répteis de muitas formas, como por exemplo: espoliação sanguínea, destruição de tecidos, competição por nutrientes, degradação de células, obstrução de vias e canais, produção de toxinas e reações alérgicas. Sendo assim, o estudo sobre os parasitos destes animais permite avaliar o seu estado de saúde, além de auxiliar na compreensão dos seus padrões de deslocamento, comportamento, distribuição e alimentação (Mackenzie, 2002).

Estudos brasileiros envolvendo aspectos relacionados à fauna parasitária de $C$. mydas são escassos. Dessa forma, é de extrema relevância que mais estudos sejam realizados, pois o conhecimento sobre aspectos ecológicos e epidemiológicos envolvendo comunidades parasitárias podem fornecer informações sobre a biologia de processos parasitários e sua relação com os hospedeiros.

Nesse contexto, objetivou-se realizar o levantamento dos helmintos gastrintestinais encontrados em exames anatomopatológicos e coproparasitológicos realizados em C. mydas (tartarugas-verdes) resgatadas no litoral sul de São Paulo, Brasil, e avaliar os aspectos ecológicos e epidemiológicos envolvidos, correlacionando os resultados com a bibliografia existente, no sentido de contribuir para o entendimento da fauna parasitária das tartarugas-verdes que visitam a costa brasileira. 


\section{MATERIAL E MÉTODOS}

Foi realizado um estudo individuado, observacional, transversal, retrospectivo, descritivo e quantitativo, onde foram analisados 118 registros de laudos de exames parasitológicos e anatomopatológicos finalizados e validados de C. mydas, que vieram a óbito ou já foram encontradas mortas, resgatadas no litoral sul de São Paulo, Brasil, realizados pelo Instituto Biopesca, no período de julho de 2018 a julho de 2019 e disponibilizados online no Sistema de Informação de Monitoramento da Biota Aquática (SIMBA).

Assim que os exames são finalizados e validados, os resultados são registrados em laudos na plataforma online (SIMBA), sendo os dados disponibilizados para acesso público. As informações encontradas foram devidamente analisadas e os dados foram

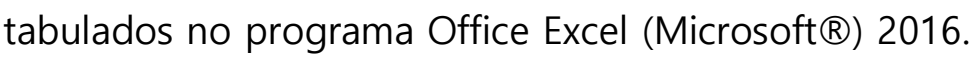

Para o levantamento dos helmintos gastrintestinais de C. mydas, obteve-se o número de animais parasitados, espécies de helmintos gastrintestinais encontrados, o órgão parasitado e a presença de mono ou poliparasitismo.

Adicionalmente, foram calculadas a prevalência, intensidade média e abundância média (Bush et al., 1997).

\section{RESULTADOS e DISCUSSÃO}

O Instituto Biopesca, localizado em Praia Grande (SP), é responsável pelo resgate de animais marinhos, como as tartarugas-verdes. Os Projetos de Monitoramento de Praias (PMP), do qual o Biopesca faz parte, são desenvolvidos para o atendimento de condicionantes do licenciamento ambiental federal, conduzido pelo IBAMA, das atividades de exploração e produção (E\&P) de petróleo e gás natural offshore da PETROBRAS nas Bacias de Santos, de Campos, do Espírito Santo, de Sergipe-Alagoas e Potiguar.

Quando deslocados à instituição, os espécimes são submetidos à exames diversos, incluindo parasitológico, coproparasitológico e anatomopatológico. Caso um indivíduo resgatado chega vivo à base de reabilitação o exame coproparasitológico é realizado para admissão do animal. Em contrapartida, quando a tartaruga chega já em óbito, a carcaça é encaminhada à necropsia que é realizada de acordo ao proposto por Wyneken (2001). Quando parasitos são encontrados, são devidamente coletados, conforme Amato et al. (1991), e enviados para análise em laboratório terceirizado. 
Foram realizados 504 registros de tartarugas marinhas no período de junho de 2018 a junho de 2019 pelo Instituto Biopesca. Dentre as tartarugas-verdes recebidas, 118 $(23,41 \%)$ foram submetidas à exames anatomopatológicos e parasitológicos. Todos (100\%) os animais registrados eram juvenis, o que também foi descrito por Gomes et al. (2017), Binote et al. (2015) e Tourinho et al. (2010). A prevalência de indivíduos neste estágio de vida pode ser associada aos hábitos alimentares da espécie, que se alimenta de algas e grama marinha ao longo da região costeira (Awabdi; Siciliano; Beneditto, 2013)

Entre as C. mydas submetidas à exames, 102 foram diagnosticadas como positivas para helmintos gastrintestinais, revelando uma prevalência de $86,44 \%$ de animais parasitados. Tal resultado coincide ao encontrado por Gomes e colaboradores (2017) no Espírito Santo, com 94,44\% de tartarugas parasitadas. Embora um estudo conduzido por Binote e colaboradores (2015), também no Espírito Santo, tenha demonstrado uma prevalência de 50,00\% de tartarugas parasitadas por helmintos e Werneck (2011), ao estudar todo o litoral brasileiro, relatou um total de 50,70\% de tartarugas parasitadas. Essa diferença de percentual de parasitismo encontrado em C. mydas, pode estar relacionada ao número amostral, visto que amostras maiores permitem uma possível obtenção de maior quantidade de animais não parasitados de maior quantidade de animais não parasitados maior quantidade de animais não parasitados.

Um total de 3.474 parasitos foram apontados pelos exames. E os órgãos mais parasitados foram intestino delgado $(63,06 \%)$ e estômago $(63,06 \%)$, seguidos de intestino grosso (0,9\%). Esses dados coincidem com o descrito por Gomes e colaboradores (2017) com as mesmas porções do trato gastrointestinal como locais mais acometidos.

Entre os animais parasitados, 61 (51,69\%) apresentavam poliparasitismo, ou seja, associação de duas até oito espécies. Foram encontrados 17 animais albergando duas espécies (14,41\%), 16 com três espécies (13,56\%), oito tartarugas-verdes com quatro espécies (6,78\%), quatro tartarugas-verdes com cinco tartarugas-verde com cinco espécies (3,39\%), dois com seis (1,69\%), um com sete $(0,85 \%)$, dois com oito $(1,69 \%)$ e 11 (9,32\%) animais foram descritos com associação, porém não apresentam nos relatórios a quantidade de parasitos associados. Outros trabalhos revelaram um valor maior, com $94,12 \%$ e $100 \%$ de animais poliparasitados (Gomes et al., 2017; Santoro et al., 2006) e a literatura demonstra que a presença de mais de uma espécie de parasitos coexistindo é normal (Matteazzi et al., 2002). 
Foram observados 50 (42,37\%) laudos de tartarugas-verdes apresentando monoparasitismo, e sete (5,93\%) indivíduos não tinham nenhum tipo de informação quanto a poliparasitismo ou monoparasitismo.

Os resultados referentes à helmintofauna podem ser observados na Tabela 1. Foram descritas 13 espécies de helmintos (Tabela 1), todos pertencentes à Classe Trematoda, indo de acordo ao estudo de Werneck (2007) que também descreveu apenas trematódeos. O número de helmintos por hospedeiro variou de 2 a 1400 e o valor para abundância média total foi de 29,44. Tal índice, quando mensurado por espécie, indica que o helminto mais abundante foi Cricocephalus albus (Kuhl \& Van Hasselt, 1822) (11,86), seguido por Metacetabulum invaginatum (Teixeira de Freitas \& Lent, 1938) $(11,61)$ e Pronocephalus obliquus (Looss, 1899) $(4,50)$.

Tabela 1 - Helmintofauna e índices ecológicos e epidemiológicos do parasitismo em Chelonia mydas resgatadas no litoral sul de São Paulo, Brasil, entre julho de 2018 e julho de 2019.

\begin{tabular}{|c|c|c|c|c|c|c|}
\hline \multicolumn{7}{|c|}{ Prevalência, abundância e intensidade médias e local de parasitismo da helmintofauna de } \\
\hline Classe & Família & Espécies & 1P (\%) & ${ }^{2} \mathrm{AM}$ & 3IM & $\begin{array}{l}\text { Local de } \\
\text { Parasitismo }\end{array}$ \\
\hline \multirow[t]{13}{*}{ Trematoda } & \multirow[t]{4}{*}{ Calycodidae } & Calycodes anthos & 1,96 & 0,042 & & $5 \mid D$ \\
\hline & & Cricocephalus albus & 75,49 & 11,86 & 18,18 & ${ }^{4} \mathrm{EST} /{ }^{6} \mathrm{IG} / \mathrm{ID}$ \\
\hline & & Pleurogonius lineares & 4,90 & 0,25 & & \\
\hline & & Pleurogonius longiusculus & 3,92 & 0,05 & 1,75 & ID \\
\hline & \multirow[t]{4}{*}{ Pronocephalidae } & Pronocephalus obliquus & 28,43 & 4,50 & 18,34 & $\mathrm{EST} / \mathrm{ID}$ \\
\hline & & Pronocephalus minutus & 0,98 & 0,01 & 2 & ID \\
\hline & & $\begin{array}{l}\text { Metacetabulum } \\
\text { invaginatum }\end{array}$ & 38,73 & 11,61 & 31,88 & $\mathrm{EST} / \mathrm{ID}$ \\
\hline & & Deuterobaris intestinalis & 0,98 & 0,05 & 6 & ID \\
\hline & \multirow[t]{2}{*}{ Microscaphidiidae } & Deuterobaris proteus & 1,96 & 0,23 & 14 & ID \\
\hline & & Polyangium linguatula & 2,94 & 0,11 & 4,33 & ID \\
\hline & Plagiorchiidae & Enodiotrema megachondrus & 12,74 & 0,22 & 2,07 & ID \\
\hline & Spirorchiidae & Learedius learedi & 1,96 & - & - & EST/ID \\
\hline & Telorchiidae & Orchidasma amphiorchis & 4,90 & 0,43 & 10,2 & IG/ID/EST \\
\hline
\end{tabular}

${ }^{1} \mathrm{P}=$ Prevalência; ${ }^{2} \mathrm{AM}=$ Abundância Média; ${ }^{3} \mathrm{IM}=$ Intensidade Média ${ }^{4} \mathrm{EST}=$ Estômago; ${ }^{5} \mathrm{ID}=$ Intestino Delgado; ${ }^{6} \mathrm{IG}=$ Intestino Grosso.

Quanto a prevalência, foi encontrado: C. albus com 75,49\% (77/102), $M$. invaginatum com 42,15\% (43/102), P. obliquus com 28,43\% (29/102) e Enodiotrema megachondrus (Looss, 1899) com e 12,74\% (13/102).

Os pesquisadores Gomes et al. (2017) e Binoti et al. (2015) no Espírito Santo e Werneck (2011) em São Paulo, também encontraram C. albus, M. invaginatum e $P$. 
obliquus como espécies de helmintos mais prevalentes e abundantes. Santoro et al. (2016) na Costa Rica, embora também tenha descrito algumas espécies deste estudo, revelou Learedius learedi, Microscaphidium reticulare e Pyelosomum cochlear como os parasitas mais abundantes.

Tal fato pode ser provavelmente justificado pela região geográfica da pesquisa influenciar na distribuição dos parasitos, visto que estes podem se adaptar a condições específicas do ambiente e disponibilidade de hospedeiros intermediários. Ainda, outro fator que poderia justificar é que as tartarugas realizam migrações de acordo a fase de vida que se encontram, assim, a sua comunidade de endoparasitos pode ser alterada de acordo ao seu deslocamento e encontro com tais parasitos.

Ainda foram encontrados em ordem decrescente de prevalência: Orchidasma amphiorchis (Braun, 1901) e Pleurogonius lineares (Looss, 1901) com 4,90\% (5/102) cada um, Pleurogonius longiusculus (Looss, 1901) com 3,92\% (4/102) e Polyangium linguatula (Looss, 1899) com 2,94\% (3/102). As espécies Calycodes anthos (Braun, 1899) Looss, 1901. Deuterobaris proteus (Brandes, 1891; Looss, 1902) e Learedius learedi (Price, 1934) apresentaram percentuais de 1,96\% (2/102) cada. E, por fim, com 0,98\% (1/102) para cada espécie, foram encontradas Deuterobaris intestinalis (Mehrotra, 1973) e Pronocephalus minutus (Ruiz, 1946)

Todas as outras espécies de helmintos encontradas, também foram descritas por Werneck e Silva (2016) em C. mydas. Algumas das espécies encontradas neste presente estudo são específicas de $C$. mydas, como: $D$. intestinalis, $D$. proteus e $P$. longiusculus.

Quanto à intensidade média, este estudo encontrou $M$. invaginatum, $C$. albus e $P$. obliquus com 31,88, 18,18 e 18,34, respectivamente. Os parasitas $M$. invaginatum e $C$. albus também foram descritos por Gomes et al. (2017) e Werneck (2011) com maiores valores de intensidade média. Assim, com os resultados encontrados, percebe-se que tais espécies são habitualmente encontradas parasitando tartarugas-verdes.

Parasitos muitas vezes possuem um ciclo biológico complexo, dependendo de interações tróficas para sua transmissão. Cada espécie de parasito reflete a presença de diferentes organismos que participam de seu ciclo de vida; em conjunto, todas as espécies de parasitos num hospedeiro refletem a presença de uma infinidade de organismos hospedeiros e interações tróficas no ambiente. Sendo assim, parasitos podem ser potencialmente utilizados como indicadores da diversidade de espécies e ecossistemas (Marcogliese, 2008).

Conforme os resultados demonstrados e comparando-os com a literatura existente, há uma semelhança na constituição da parasitofauna das C. mydas ao longo da 
costa brasileira, sendo as espécies de helmintos $C$. albus, $M$. invaginatum e $P$. obliquus as mais comuns. O presente estudo apresentou a comunidade de endoparasitas de tartarugas juvenis e pode ser inferido que a fase de vida das tartarugas possivelmente influencia na comunidade de helmintos tanto pelo estresse gerado na passagem de filhote para juvenil com uma mudança drástica em sua alimentação, quanto mudança de habitat de ambiente oceânico para costeiro. Além disso, os resultados demonstram que a prevalência, intensidade e abundância média são altos quando equiparadas com os estudos consultados, indicando que a parasitofauna em tartaugas-verdes juvenis pode ser prevalente, variada e abundante. O resultado encontrado pode ser sugerido por essa espécie, durante a fase de vida juvenil, não realizar intensas migrações e apresentar hábito costeiro, aumentando a chance dos helmintos de encontrarem hospedeiros intermediários. Além disso, algas podem servir de alimento para uma variada quantidade de espécies, dessa forma, para potenciais hospedeiros intermediários.

\section{CONCLUSÃO}

Os helmintos gastrintestinais de Chelonia mydas resgatados no litoral sul de São Paulo são constituídos predominantemente de trematódeos, com elevada prevalência, intensidade e abundância média quando comparados à literatura existente.

\section{CONSIDERAÇÕES FINAIS}

É evidente a necessidade das pesquisas relacionadas à área de parasitologia das espécies $C$. mydas, sendo, portanto, um campo fértil para se fazer ciência e contribuir com o enriquecimento do conhecimento existente. Isso porque, permite o entendimento e mensuração dos impactos humanos sobre a espécie e as suas relações ecológicas, além de permitir que a conservação das C. mydas e outras tartarugas marinhas possa ser aplicada e aprimorada, visto que os parasitos de uma espécie também podem servir como bioindicadores.

\section{Agradecimento}

Os autores agradecem ao Instituto Biopesca e a Petrobras pelo apoio para o desenvolvimento de pesquisas com a fauna marinha da região.

\section{REFERÊNCIAS}


AWABDI, D. R.; SICILIANO, S.; DI BENEDITTO, A.P.M. Ingestão de resíduos sólidos por tartarugas-verdes juvenis, Chelonia mydas (L. 1758), na costa leste do estado do Rio de Janeiro, Brasil. Biotemas 26: 197-200. 2013

BINOTI, E.; GOMES, M.C.; CALAIS, A. Helminth fauna of Chelonia mydas (Linnaeus, 1758) in the south of the state of Espírito Santo and description of tissue injury. Helminthologia, v.53, p.195-199, 2015.

BRASIL. Ministério do Meio Ambiente. [2015]. Lista de Espécies da fauna brasileira ameaçada de extinção. Disponível em: <https://www.icmbio.gov.br/portal/biodiversidade/fauna-brasileira/lista-deespecies/6611especie6611.html>. Acesso em 13/05/2020.

BUSH, A.O.; LAFFERTY, K.D.; LOTZ, J.M. et al. Parasitology meets ecology on its own terms: Margolis et al. revisited. Journal of Parasitoly, v.83, p.575-583, 1997.

Cubas, Z. S.; Baptistotte, C. Chelonia (Tartaruga, Cágado, Jabuti). In: CUBAS, Z. S; SILVA, J. C. R; DIAS, J. L. C. Tratado de Animais Selvagens. 2. ed, São Paulo: Roca, 2014. Vol. 2, Cap. 9, p. 108-110.

ERASMUS, D. A. General features. In _. The biology of the trematodes. London, Edward Arnold Publishers, 1972. p. 1-35.

GEORGE, R.H.; MILLER, J. Health problems and diseases of sea turtles. In: LUTZ, P.L.; MUSICK, J.A. The Biology of the Sea Turtle. Boca Raton: CRC Press, 1997. Cap. 14. p.5158.

GOMES, M.C.; MARTINS, I.V.F.; WERNECK, M.R. et al. Ecologia da comunidade de helmintos gastrointestinais de tartarugas-verdes (Chelonia mydas) recolhidas no litoral do Espírito Santo. Arquivo Brasileiro de Medicina Veterinária e Zootecnia v.69, n.3, p.644650, 2017.

GREINER, E.C. Parasites of marine turtle. In: WYNEKEN, J.; LOHMANN, K.J.; MUSICK, J.A. The biology of sea turtles. Florida: CRC Press, 2013. v.3, p.425-444.

IUCN 2015. The IUCN Red List of Threatened Species. Disponível em: < https://www.iucnredlist.org/>. Acesso em 13/05/2020. 
KAMEL, S. J.; MROSOVSKY, N.; MROSOVSKY, M. Inter-Seasonal Maintenance of Individual Nest Site Preferences in Hawksbill Sea Turtles. Vol. 87, No. 11. p. 2947-2952. 2006

KLINGENBERG, R. J. Understanding reptile parasites. $1^{\text {st }} . e d$. Lakeside. Advanced Vivarium Systems, 1993. 83p

LUTCAVAGE, M. E.; LUTZ, P. L. Diving physiology. In LUTZ, P. L.; MUSICK, J. A. The biology of sea turtles. 1. ed. New York: CRC Marine Science series, 1997. p. 277-296.

MACKENZIE, K. Parasites as biological tags in population studies of marine organisms: an update. Parasitology. v.124 p.153-163. 2002.

Mackenzie, K. Parasites as indicators of host populations. International Journal of Parasitology, 17, 345-352. 2002.

MARCOGLIESE, D.J. Food webs and the transmission of parasites to marine fish. Parasitology, v.124, p.83-99, 2002.

MARCOGLIESE, D.J. The impact of climate changes in the parasites and infectious diseases of aquatic animals. Revue Scientifique et Technique. Paris, v 27, n. 2, p. 467-484. 2008.

MARCOVALDI, M. A.; MARCOVALDI, G. G. Marine turtles of Brazil: The history and structure of Projeto TAMAR-IBAMA. Biological Conservation, v.91, p.35-41, 1999.

MÀRQUEZ, R.M. Sea turtles of the world. An annotated and illustrated catalogue of sea turtle species known to date. FAO Fish. Synopsis, v.11, n.125, 81p. 1990.

MATTEAZZI, L.; SANTOS, S. M. C.; FERNANDEZ, J. S.; BECKER, J.H. Ocorrência de endohelmintos no trato digestório de tartarugas marinhas, Chelonia mydas (Linnaeus, 1758) (Testudines: Cheoniidae) encontradas mortas no litoral de Ubatuba-SP. In: ENCONTRO DE INICIAÇÃO CIENTíFICA， 7; MOSTRA DE PÓS-GRADUAÇÃO， 3, 2002, Taubaté. Anais... Taubaté: UNITAU, 2002. p.127.

NAVARRO, C. E. K. G.; PACHALY, J. R. Manual de Hematologia Veterinária. 1. ed. São Paulo: Livraria Varela, 1994. 
NEVES, D.P.; MELO, A.L.; LINARDI, P.M. et al. Parasitologia Humana. Editora Atheneu. Ed. 11a. São Paulo. 2005.

NORTON, T.; WYNEKEN, J. Body Condition Scoring the Sea Turtle. LafeberVet. 2015. Disponível em: < https://lafeber.com/vet/body-condition-scoring-the-seaturtle/\#: :text=A\%20body\%20condition\%20score\%20(BCS,6)\%20and\%205\%20is\%20obese. $>$ Acesso em: 11/08/2020.

PRITCHARD, P. C. H. Evolution, phylogeny and current status. In.: MUSICK, J.A. LUTZ. P.L.The Biology of sea turtles: 1. ed. New York: CRC Marine Science series, 1997. p.1-28.

RODENBUSCH, C. R.; MARKS, F.S.; CANAL, C. W. et al. Marine leech Ozobranchus margoi parasitizing loggerhead turtle (Caretta caretta) in Rio Grande do Sul, Brazil. Revista Brasileira de Parasitologia Veterinária. v.21 nº.3 Jaboticabal. 2012

SAAD, A. H.; EL DEEB, S. Immunological changes during pregnancy in the viviparous lizard, Chalcides ocellatus. Veterinary Immunology and Immunopathology. v. 25. p. 279-286. 1990

SANTORO, M.; GREINER, E.C.; MORALES, J.A.; RODRÍGUEZ-ORTÍZ, B. Digenetic trematode community in nesting green sea turtles (Chelonia mydas) from Tortuguero Nacional Park, Costa Rica. Journal of Parasitoly, v.92, p.1202- 1206, 2006.

SAWYER, R.T.; Leech Biology and Behaviour. Oxford University Press. 1986

SILVA, A.C.C.D. et al. Efforts to reduce sea turtle bycatch in the shrimp fishery in Northeastern Brazil through a co-management process. Ocean and Coastal Management, 53: 570-576. 2010.

SPOTILA, J. R. Sea turtles: a complete guide to their biology, behavior and conservation. Baltimore, Md.: Johns Hopkins University Press. 2004

TARTARUGA verde. Projeto Tamar. Disponível em: <http://www.tamar.org.br/tartaruga.php?cod=20> Acesso em: 14/05/2020.

TOURINHO, P.S.; SUI, J.A.I., FILLMANN, G. Is marine debris ingestion still a problem for the coastal marine biota of southern Brazil? Marine Pollution Bulletin, p. 396-401. 2010. 
WERNECK, M.R. Estudo da helmintofauna de tartarugas marinhas procedentes da costa brasileira. 2011. Botucatu, 138f. Tese (Doutorado em Biologia de parasitas e microorganismos) - Programa de Pós-Graduação em Biologia Geral e Aplicada da Universidade Estadual Paulista.

WERNECK, M.R.; SILVA, R.J. Helminth parasites of juvenile green turtles Chelonia mydas (Testudines, Cheloniidae) in Brazil. Journal of Parasitoly, v.101, p.713-716, 2016.

WYNEKEN, J. The anatomy of sea turtle. Miami: National Oceanic and Atmospheric Administration. 2001. p.33-42. 